\title{
Parallel Sequence Alignment: A Lookahead Approach
}

\author{
Prasanta K. Jana and Nikesh Kumar \\ Department of Computer Science and Engineering, \\ Indian School of Mines, Dhanbad-826004, India \\ prasantajana@yahoo.com, kumar_nikesh@yahoo.co.uk
}

\begin{abstract}
In this paper we present a parallel algorithm for local alignment of two biological sequences. Given two sequences of size $m$ and $n$, our algorithm uses a novel technique namely, carry lookahead and requires $O(m / 4+n / 2)$ time on a maximum of $O(m)$ processors.
\end{abstract}

\section{Introduction}

Sequence alignment is a very fundamental problem in modern molecular biology. Searching similarity or homologous sequences in the large biological databases through local alignment has now become a regular practice. Given two sequences $S_{1}$ and $S_{2}$, the problem of local alignment is to find two subsequences $\alpha$ and $\beta$ of $S_{1}$ and $S_{2}$ respectively whose similarity (optimal global alignment value) is maximum over all pairs of subsequences from $S_{1}$ and $S_{2}$. The Smith-Waterman algorithm [5] is an optimal solution for the local alignment problem. A more restricted case of this problem known as the local suffix alignment is as follows. Let $V(i, j)$ be defined as the optimal alignment of prefixes $S_{1}[1 \ldots i]$ and $S_{2}[1 \ldots j]$. Given a pair of indices $i \leq m$ and $j \leq n$, the problem of local suffix alignment is to find a (possibly empty) suffix $\alpha$ of $S_{1}[1 \ldots i]$ and a (possibly empty) suffix $\beta$ of $S_{2}[1 \ldots j]$ such that $V(\alpha, \beta)$ is the maximum over all pairs of suffixes of $S_{1}[1 \ldots i]$ and $S_{2}[1 \ldots j]$. Let $v(i, j)$ denote the value of the optimal local suffix alignment for the given index pair $i, j$. The dynamic programming solution for the local suffix alignment problem is based on the following recurrence relation [6]:

$$
v(i, j)=\max \left\{\begin{array}{l}
0 \\
v(i-1, j-1)+s\left(S_{1}(i), S_{2}(j)\right) \\
v(i-1, j)+s\left(S_{1}(i),-\right) \\
v(i, j-1)+s\left(S_{-}, S_{2}(j)\right)
\end{array} \text { for } i>0 \text { and } j>0\right.
$$

with $v(i, 0)=0$ and $v(0, j)=0 \forall i, j$ where $s$ denotes a scoring function and '-' denotes a space that implies an insertion or deletion. A solution to the local suffix alignment problem also solves the local alignment problem [6]. In the recent years many researchers have developed various parallel algorithms and parallel architectures for this problem which can be found in [1], [2], [3], [4],[7], [8], [9]. In this paper, we present a parallel algorithm for comparing two biological sequences through local alignment. In most of the dynamic programming based parallel algorithms, parallelism has been applied to calculate all the table elements concurrently on a single diagonal only and the computations proceed diagonal by diagonal. In contrast, our algorithm, however, computes all the table elements of four diagonals simultaneously 
using lookahead approach. This requires $O(m / 4+n / 2)$ time using a maximum of $O(m)$ processors.

\section{Proposed Parallel Algorithm}

The basic idea of our algorithm is to first divide the entire dynamic programming table row wise into two equal parts, upper and lower using Hirschberg method [10]. Then we perform the computations to fill up the entries in the upper part and the lower part of the table concurrently in forward and backward direction followed by trace backs in both the parts and then combine the results of the trace backs. This is important to note that although the Hirschberg method is applicable to solve global alignment problem it can also be used for solving the local alignment problem for two sequences as explained in the section 12.1.5 of the text of Gusfield [6].

We now consider the lookahead approach for calculating the table elements for the upper part only. For symmetry, the elements of the lower part of the table can be calculated similarly. From (1.1) we have the following two equations

$$
\begin{aligned}
& v(i-1, j)=\max \left\{\begin{array}{l}
0 \\
v(i-2, j-1)+s\left(S_{1}(i-1), S_{2}(j)\right) \\
v(i-2, j)+s\left(S_{1}(i-1),-\right) \\
v(i-1, j-1)+s\left(+, S_{2}(j)\right)
\end{array}\right. \\
& \text { and } \quad v(i, j-1)=\max \left\{\begin{array}{l}
0 \\
v(i-1, j-2)+s\left(S_{1}(i), S_{2}(j-1)\right) \\
v(i-1, j-1)+s\left(S_{1}(i),-\right) \\
v(i, j-2)+s\left(-, S_{2}(j-1)\right)
\end{array}\right.
\end{aligned}
$$

Substituting $v(i-1, j)$ and $v(i, j-1)$ from (2.1) and (2.2) in equation (1.1), we obtain

$$
v(i, j) \leftarrow \max \left\{\begin{array}{l}
0 \\
v(i-1, j-1)+s\left(S_{1}(i), S_{2}(j)\right. \\
\left.v(i-1, j-1)+s\left(S_{2}, j\right)\right)+s\left(S_{1}(i), S_{-}\right) \\
v(i-2, j-1)+s\left(S_{1}(i-1), S_{2}(j)\right)+s\left(S_{1}(i),{ }_{-}\right) \\
v(i-2, j)+s\left(S_{1}(i-1),{ }_{-}\right)+s\left(S_{1}(i),,_{-}\right), \\
v(i-1, j-2)+s\left(S_{1}(i), S_{2}(j-1)\right)+s\left({ }_{-}, S_{2}(j)\right) \\
v(i, j-2)+s\left({ }_{-}, S_{2}(j-1)\right)+s\left({ }_{-}, S_{1}(j)\right)
\end{array}\right.
$$

From the dependency graph (composite) as shown in Fig. 1, it is clear that computations of all three elements, i.e., $v(\underline{i, j}), v(i-1, j)$ and $v(i, j-1)$ are independent of each others. Therefore we can compute $v(i-1, j)$ and $v(i, j-1)$ using equation (1.1) from the values of the just two previous diagonals along with $v(i, j)$ using equation (2.3) from the values of the previous to previous two diagonal elements for all possible values of $i$ and $j$. It means that all the elements of two consecutive diagonals (shown by solid lines in this figure) can be calculated concurrently. Since the table elements of the lower part will be calculated in the reverse direction, we replace 'symbol by ' + ' for all the indices of $v(i, j)$ in equations (1.1) and (2.3).

We now formally present the parallel algorithm stepwise as follows. Since computing the table elements is the dominating one, in our parallel version, we only consider the generation of the entire table elements. 


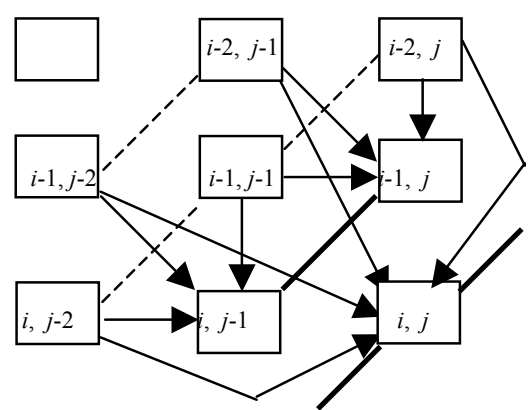

Fig. 1. Dependency graph of computing $v(i-1, j), v(i, j-1)$ and $v(i, j)$

\section{Algorithm Par_alignment:}

1. do steps 2 and 3 in parallel

2. for diag $=2$ to $m / 2+n$ by step 2

for all $i, j, i=1$ to $m / 2$ and $j=1$ to $n$ do steps 2.1 and 2.2 in parallel

2.1 if $(i+j=$ diag $)$ then

$$
v(i, j) \leftarrow \max \left\{\begin{array}{l}
0, v(i-1, j-1)+s\left(S_{1}(i), S_{2}(j)\right) \\
v(i-1, j)+s\left(S_{1}(i),-\right) \\
v(i, j-1)+s\left({ }_{-}, S_{2}(j)\right)
\end{array}\right.
$$

2.2 if $(i+j=\operatorname{diag}+1)$ then

$$
v(i, j) \leftarrow \max \left\{\begin{array}{l}
0, v(i-1, j-1)+s\left(S_{1}(i), S_{2}(j)\right. \\
v(i-1, j-1)+s\left({ }_{-}, S_{2}(j)\right)+s\left(S_{1}(i),-\right) \\
v(i-2, j-1)+s\left(S_{1}(i-1), S_{2}(j)\right)+s\left(S_{1}(i),-\right) \\
v(i-2, j)+s\left(S_{1}(i-1),-\right)+s\left(S_{1}(i),-\right), \\
v(i-1, j-2)+s\left(S_{1}(i), S_{2}(j-1)\right)+s\left({ }_{-}, S_{2}(j)\right) \\
v(i, j-2)+s\left({ }_{-}, S_{2}(j-1)\right)+s\left(\ldots, S_{1}(j)\right)
\end{array}\right.
$$

\section{3. for $\operatorname{diag}=2$ to $m / 2+n$ by step 2}

forall $i, j, i=m$ downto $m / 2+1$ and $j=n$ downto 1 do steps 3.1 and 3.2 in parallel

3.1 if $(i+j-2+\operatorname{diag}=m+n)$ then

$$
v(i, j) \leftarrow \max \left\{\begin{array}{l}
0, v(i+1, j+1)+s\left(S_{1}(i), S_{2}(j)\right) \\
v(i+1, j)+s\left(S_{1}(i),-\right) \\
v(i, j+1)+s\left({ }_{-}, S_{2}(j)\right)
\end{array}\right.
$$

3.2 if $(i+j-2+\operatorname{diag}=m+n-1)$ then

$$
v(i, j) \leftarrow \max \left\{\begin{array}{l}
0, v(i+1, j+1)+s\left(S_{1}(i), S_{2}(j)\right) \\
v(i+1, j+1)+s\left(\bar{S}_{2}(j)\right)+s\left(S_{1}(i),{ }_{-}\right) \\
v(i+2, j+1)+s\left(S_{1}(i+1), S_{2}(j)\right)+s\left(S_{1}(i),{ }_{-}\right) \\
v(i+2, \mathrm{j})+s\left(S_{1}(i+1),{ }\right)+s\left(S_{1}(i),{ }_{-}\right) \\
v(i+1, j+2)+s\left(S_{1}(i), S_{2}(j+1)\right)+s\left({ }_{-}, S_{2}(j)\right) \\
v(i, j+2)+s\left({ }_{-}, S_{2}(j+1)\right)+s\left(\ldots, S_{1}(j)\right)
\end{array}\right.
$$

4. Stop 
Complexity: The steps 2 and 3 run in parallel each of which iterates $m / 4+n / 2$ times and the other steps require constant time. So the above algorithm requires $O(\mathrm{~m} /$ $4+n / 2)$ time. Since the maximum size of the diagonal of the upper or lower table is $m / 2$, it requires maximum $2 m$ processors to compute four diagonals in parallel.

\section{Conclusion}

A parallel algorithm has been presented for local alignment of two biological sequences based on lookahead approach. Given two sequences of size $m$ and $n$ respectively, our algorithm computes the dynamic programming table diagonal by diagonal requiring $O(m / 4+n / 2)$ time and a maximum of $O(m)$ processors.

\section{References}

1. Huang, X.: A space efficient parallel sequence comparison algorithm for message-passing multiprocessors. International J. of Parallel Programming. 18 (1989) 223-239.

2. Lander, E., Mesirov, J., Taylor, W.: Protein sequence comparison on a data parallel computer. Proceedings of the 1988 International Conference on Parallel Processing (1988) 257-263.

3. Aluru, S., Futamura, N., Mehrotra, K.: Parallel biological sequence comparison using prefix computations. J. of Parallel and Distributed Computing. 63 (2003) 264 -272.

4. Edmiston, E., Core, N., Saltz, J., Smith, R.: Parallel processing of biological sequence comparison algorithms. International J. of Parallel Programming. 17 (1988) 259-275.

5. Smith, T. F., Waterman, M. S.: Identification of common molecular subsequences. J. of Molecular Biology. 147 (1981) 195-197.

6. Gusfield, D.: Algorithms on Strings Trees and Sequences. Cambridge University Press (1997).

7. Bokhari, S. H., Saurer, J. R.: Sequence alignment on the Cray MTA-2. Proceedings of Second IEEE International Workshop on High Performance Computational Biology, April 22, Nice, France (2003).

8. Schmidt, B., Schroder, H., Schimmler, M.: Massively Parallel Solutions for Molecular Sequence Analysis. Proceedings of $1^{\text {st }}$ International workshop on High Performance Computational Biology (2002).

9. Martins, W. S., del Cuvillo, J., Cui, W., Gao, G., Whole genome alignment using a multithreaded parallel implementation. Proceedings of Symposium on Computer Architecture and High Performance Computing, Pirenopolis, Brazil, (2001) 1-8.

10. Hirschberg, D. S.: Algorithms for the longest common subsequence problem. J. ACM 24 ( 1977) 664-675. 